\title{
Minimal sets on tori
}

\author{
DANIEL BEREND \\ Department of Mathematics, University of California, Los Angeles, CA 90024, USA
}

(Received 12 January 1984)

Abstract. Let $\Sigma$ be a commutative semigroup of continuous endomorphisms of the $r$-dimensional torus. Generalizing a result of Furstenberg dealing with the circle group, necessary and sufficient conditions are given here for $\Sigma$ to possess the following property: Any $\Sigma$-minimal set consists of torsion elements. Semigroups not having this property are shown to admit minimal sets of positive Hausdorff dimension.

\section{Introduction}

In topological dynamics one usually studies a flow $(\Omega, S)$, composed of a compact Hausdorff space $\Omega$ and a semigroup $S$ of continuous transformations thereof. Closed $S$-invariant subsets of $\Omega$ give rise to subflows of $(\Omega, S)$. Of particular importance are the minimal subflows of $\Omega$.

Flows formed of semigroups of endomorphisms of compact abelian groups play a special role and were analyzed from various points of view. Such a flow cannot be minimal, but may have weaker properties of a similar nature. For example, it may be ergodic, which amounts to every invariant proper subset of the group being of the first category. In [3] Furstenberg examined, for the case of the circle group $\mathbb{T}$, several properties which lie between ergodicity and minimality. Semigroups of endomorphisms of $\mathbb{T}$ correspond to multiplicative semigroups of integers. Calling such a semigroup lacunary if all its positive elements are powers of a single integer and non-lacunary otherwise, some of Furstenberg's results [3, prop. III.1, th. III.2, prop. IV.2, th. IV.1] can be combined to yield the following:

THEOREM. Let $\Sigma$ be a semigroup of epimorphisms of $\mathbb{T}$. The following conditions are equivalent:

(1) Every closed $\Sigma$-invariant proper subset of $\mathbb{T}$ is a finite set of torsion elements.

(2) Every $\Sigma$-minimal set consists of torsion elements.

(3) $\Sigma$ is ergodic and every $\Sigma$-minimal set is finite.

(4) $\Sigma$ is ergodic and every $\Sigma$-minimal set is of Hausdorff dimension 0.

(5) $\Sigma$ is non-lacunary.

We mention that the theorem is interesting from the point of view of the theory of diophantine approximations also. It implies in particular a well-known theorem of Hardy and Littlewood [6] according to which if $\alpha$ is an irrational number and $k$ a positive integer then the set $\left\{n^{k} \alpha \mid n \in \mathbb{N}\right\}$ is dense modulo 1 . This aspect is discussed in detail in [3] and [4]. 
It can now be asked under what conditions a commutative semigroup $\Sigma$ of epimorphisms of $\mathbb{T}^{r}$ has any of the properties (1)-(4) of the theorem. In [1] this question was settled for property (1). It is easy to see that the following three properties are strictly weaker if $r>1$. The main result of this paper is the equivalence of these properties and a full characterization of those semigroups possessing them. The conditions under which a semigroup has this property are presented in $\S 2$.

$\S 3$ deals with proving the necessity of the conditions. Namely, given any commutative semigroup for which those conditions are violated, we construct a minimal set of positive Hausdorff dimension.

In $\S 4$ we prove the sufficiency of the conditions, i.e. that if a given semigroup satisfies the conditions in question then any minimal set is composed of torsion elements. First the results of [3] and [1] are employed to handle a special, 'totally irreducible' case. The general case is then reduced to this one by means of appropriate decompositions of $\mathbb{T}^{r}$ and corresponding decompositions of the acting semigroup.

\section{The principal theorem}

$\mathbb{T}^{r}$ will denote the $r$-dimensional torus, considered as an additive group: $\mathbb{T}^{r}=\mathbb{R}^{r} / \mathbb{Z}^{r}$. Points of $\mathbb{T}^{r}$ are column $r$-vectors. Continuous endomorphisms of $\mathbb{T}^{r}$ are represented by $r \times r$ matrices with integer entries. Points and endomorphisms of $T^{r}$ can be lifted to points and to linear transformations of $\mathbb{R}^{r}$, respectively.

Let $\Sigma$ be a semigroup of endomorphisms of $\pi^{r}$. Put $\Sigma A=\bigcup_{\sigma \in \Sigma} \sigma(A)$ for $A \subseteq \pi^{r}$. A set $E$ is $\Sigma$-invariant if $\Sigma E \subseteq E$. A closed $\Sigma$-invariant set $M$ is $\Sigma$-minimal if it contains no closed $\Sigma$-invariant proper subset.

Definition 2.1. A semigroup of endomorphisms $\Sigma$ of a compact abelian metric group $G$ is a (i) $M H_{0}$, (ii) $M F$, (iii) $M T$ semigroup if any $\Sigma$-minimal set is (i) of Hausdorff dimension 0 , (ii) finite, (iii) composed of torsion elements, respectively.

Our interest in this paper is in characterizing the commutative $M T$ semigroups of epimorphisms of $\pi^{r}$. To present this characterization several notations and definitions are required. evec $\Sigma$ denotes the set of common eigenvectors of $\Sigma$ lying in $\mathbb{C}^{r}$. If $v \in \operatorname{evec} \Sigma$ then $\operatorname{spec}_{v} \Sigma$ is the set of eigenvalues corresponding to $v$ of all the endomorphisms in $\Sigma$. $\mathbb{C}_{1}$ denotes the group of complex numbers of modulus $1 . \Sigma$ is called hyperbolic if $\operatorname{spec}_{v} \Sigma \nsubseteq \mathbb{C}_{1}$ for each $v \in \operatorname{evec} \Sigma$. Two elements $\alpha$ and $\beta$ of a ring $R$ are rationally dependent if $\alpha^{l}=\beta^{m}$ for some integers $l$ and $m$, not both of which are 0 , and rationally independent otherwise. A subset of $R$ is one-parameter if all of its elements are powers of a single element and weakly one-parameter if any two of its elements are rationally dependent. $\Sigma$ is weakly one-parameter in some direction if $\operatorname{spec}_{v} \Sigma$ is weakly one-parameter for some $v \in$ evec $\Sigma$ and multi-parameter otherwise. $\Sigma$ is ergodic if every $\Sigma$-invariant measurable set is of (Haar) measure 0 or 1 . Now we can state the main theorem.

THEOREM 2.1. Let $\Sigma$ be a commutative semigroup of epimorphisms of $\pi^{r}$. The following conditions are equivalent:

(1) $\Sigma$ is an MT semigroup.

(2) $\Sigma$ is an ergodic $M F$ semigroup.

(3) $\Sigma$ is an ergodic $M H_{0}$ semigroup. 
(4) $\Sigma$ is hyperbolic and multi-parameter.

Remark 2.1. Slight modifications in the proof of the theorem enable one to obtain a full characterization of commutative $M T$ semigroups of arbitrary endomorphisms of $\mathbb{T}^{r}$. It turns out that $\Sigma$ is $M T$ iff for every $v \in$ evec $\Sigma$ either (i) $0 \in \operatorname{spec}_{v} \Sigma$, or (ii) $\operatorname{spec}_{v} \Sigma$ is not contained in $\mathbb{C}_{1}$ and is not weakly one-parameter. We shall not engage in proving this version.

The implication $(2) \Rightarrow(3)$ is clear. Let us show that $(1) \Rightarrow(2)$. Suppose $\Sigma$ is $M T$. In view of $[2$, th. 6.1$]$ it is sufficient to show that every finite $\Sigma$-invariant set consists of torsion elements. Let $F$ be a finite $\Sigma$-invariant set and $x$ an arbitrary element of $F$. Take a $\Sigma$-minimal set $M \subseteq \Sigma x$. Since $\Sigma$ is $M T$ every element of $M$ is a torsion element. It follows that $\sigma(x)$ is a torsion element for some $\sigma \in \Sigma$. Thus $\sigma(l x)=$ $l \sigma(x)=0$ for some $l \in \mathbb{N}$, whence by [1, lemma 5.1] $l x$ is a torsion element, and so $x$ is a torsion element as well, which proves the required implication.

It remains to prove the implications $(3) \Rightarrow(4)$ and $(4) \Rightarrow(1)$. These will be termed 'the necessity of the conditions' and 'the sufficiency of the conditions', respectively. The first will be proved in the next section and the second in $\S 4$.

\section{The necessity of the conditions}

Throughout this section $\Sigma$ denotes a commutative ergodic $M H_{0}$ semigroup of epimorphisms. We have to show that $\Sigma$ is hyperbolic and multi-parameter. This amounts to some assertions regarding the sets $\operatorname{spec}_{v} \Sigma, v \in \operatorname{evec} \Sigma$. Denoting by $\mathbb{Q}(A)$ the field extension of $\mathbb{Q}$ obtained by adjoining the set $A$ to it, we may restrict our attention to vectors $v$ with $v \in \mathbb{Q}\left(\operatorname{spec}_{v} \Sigma\right)^{r}$. In fact, if $v \in \operatorname{evec} \Sigma$ then there exists a vector $v^{\prime} \in \mathbb{Q}\left(\operatorname{spec}_{v} \Sigma\right)^{r}$ such that $\sigma v=\lambda v$ implies $\sigma v^{\prime}=\lambda v^{\prime}$, and in particular $\operatorname{spec}_{v^{\prime}} \Sigma=\operatorname{spec}_{v} \Sigma$. Consequently from now on, given a vector $v \in$ evec $\Sigma$, it will be implicitly assumed that $v \in \mathbb{Q}\left(\operatorname{spec}_{v} \Sigma\right)^{r}$.

LEMMA 3.1. $\Sigma$ is hyperbolic.

Proof. Assume, to the contrary, that $\operatorname{spec}_{v} \Sigma \subseteq \mathbb{C}_{1}$ for some $v \in \operatorname{evec} \Sigma$. Then also $\bar{v} \in \operatorname{evec} \Sigma$ and if $\sigma v=\lambda v$ then $\sigma \bar{v}=\overline{\lambda v}$, where the bar denotes complex conjugation. The projection in $\mathbb{J}^{r}$ of the ellipse

$$
\mathscr{E}_{\rho}=\left\{\alpha v+\overline{\alpha v} \mid \alpha \in \rho \mathbb{C}_{1}\right\} \subseteq \mathbb{R}^{r}
$$

is a closed $\Sigma$-invariant set of dimension 1 for any $\rho>0$. Since $\Sigma$ is ergodic there exists an ergodic $\sigma \in \Sigma[2$, th. 5.1]. Suppose $\sigma v=\lambda v$. Since $\sigma$ is ergodic $\lambda$ is not a root of unity, so that the sequence $\left(\lambda^{n}\right)_{n=0}^{\infty}$ is dense in $\mathbb{C}_{1}$. It follows that $\mathscr{E}_{\beta}$ is $\sigma$-minimal and therefore certainly $\Sigma$-minimal. The contradiction proves the lemma.

The Hausdorff dimension of a subset $A$ of a given metric space $(X, d)$ will be denoted by $\operatorname{dim} A$.

LemMA 3.2. Let $(X, d)$ and $(Y, d)$ be metric spaces and $\psi$ a mapping of $X$ onto $Y$ satisfying

$$
d\left(\psi\left(x_{1}\right), \psi\left(x_{2}\right)\right) \leq C d\left(x_{1}, x_{2}\right), \quad x_{1}, x_{2} \in X,
$$

for some $C$. Then $\operatorname{dim} Y \leq \operatorname{dim} X$. 
The proof is straightforward.

\section{LEMMA 3.3. $\Sigma$ is not a one-parameter semigroup.}

Proof. Given a matrix $\sigma$ we denote by $S(\sigma)$ the semigroup consisting of all nonnegative powers of $\sigma$ and by $G(\sigma)$ the group of all integral powers of $\sigma$. If $\sigma$ is an automorphism of $\pi^{r}$ then any $S(\sigma)$-minimal set is $G(\sigma)$-invariant, so that it suffices to show that $\Sigma$ is not a semigroup of the form $S(\sigma)$. Assume, to the contrary, that $\Sigma=S(\sigma)$ for some epimorphism $\sigma$. To arrive at a contradiction, an $S(\sigma)$-minimal set of positive Hausdorff dimension has to be constructed. In view of lemma 3.1 we may assume that $\sigma$ is hyperbolic.

Select an eigenvalue $\lambda_{1}$ of $\sigma$ and a corresponding eigenvector $v^{(1)} \in \mathbb{Q}\left(\lambda_{1}\right)^{r}$. We may assume all the components of $v^{(1)}$ to be algebraic integers. The conjugations of $\mathbb{Q}\left(\lambda_{1}\right)$ give rise to eigenvalues $\lambda_{2}, \ldots, \lambda_{s}$ of $\sigma$ and corresponding eigenvectors $v^{(2)}, \ldots, v^{(s)}$. The vector $e=\sum_{i=1}^{s} v^{(i)}$ is a non-zero element of $\mathbb{Z}^{r}$.

Suppose first that $\left|\lambda_{i}\right|>1,1 \leq i \leq s$. We may assume $\min _{1 \leq i \leq s}\left|\lambda_{i}\right|$ to be arbitrarily large. In fact, if $M$ is $\sigma^{l}$-minimal then $\bigcup_{i=0}^{l-1} \sigma^{i}(M)$ is $\sigma$-minimal. Take a positive integer with $4<a<\min _{1 \leq i \leq s}\left|\lambda_{i}\right|$ and put $\Delta=\{0,1, \ldots, a-1\}^{\mathbb{N}}$. Let $T$ denote the shift on $\Delta$. As in the proof of theorem III.2 in [3] we can construct a $T$-minimal set $M \subseteq \Delta$ having the following property: The number $B_{n}$ of distinct blocks of length $n$ occurring in any point of $M$ grows exponentially with $n$. Define a function $\psi: \Delta \rightarrow \mathbb{R}^{r}$ by:

$$
\psi(\xi)=\sum_{n=1}^{\infty} \xi(n) \sigma^{-n}(e)=\sum_{i=1}^{s}\left(\sum_{n=1}^{\infty} \xi(n) \lambda_{i}^{-n}\right) v^{(i)}, \quad \xi \in \Delta .
$$

Set $M_{1}=\psi(M)$. The projection of $M_{1}$ in $\pi^{r}$, to be denoted by $M_{2}$, is obviously $\sigma$-minimal. We claim that $\operatorname{dim} M_{2}>0$. Since in general $\operatorname{dim} \bigcup_{i=1}^{k} A_{i}=$ $\max _{1 \leq i \leq k} \operatorname{dim} A_{i}$ we have $\operatorname{dim} M_{2}=\operatorname{dim} M_{1}$. Let $\tilde{M}_{1}$ be the set of vectors of coefficients of all points in $M_{1}$ with respect to any basis of $\mathbb{C}^{r}$ whose $s$ initial elements are $v^{(1)}, v^{(2)}, \ldots, v^{(s)}$. By lemma 3.2 we have $\operatorname{dim} M_{1}=\operatorname{dim} \tilde{M}_{1}$, so that it has only to be shown that $\operatorname{dim} \tilde{M}_{1}>0$. Invoking the same lemma again we find that it suffices to prove that $\operatorname{dim} \pi_{1}\left(\tilde{M}_{1}\right)>0$, where $\pi_{1}: \mathbb{C}^{r} \rightarrow \mathbb{C}$ is given by $\pi_{1}\left(z_{1}, z_{2}, \ldots, z_{r}\right)^{T}=z_{1}$. Put:

$$
\mathscr{C}_{\alpha}=\left\{\sum_{n=1}^{\infty} \xi(n) \alpha^{-n} \mid \xi \in M\right\}, \quad|\alpha|>a .
$$

With this notation $\pi_{1}\left(\tilde{M}_{1}\right)=\mathscr{C}_{\lambda_{1}}$. For $|\beta| \geq|\alpha|>a$ consider the map $i: \mathscr{C}_{\alpha} \rightarrow \mathscr{C}_{\beta}$ given by:

$$
i\left(\sum_{n=1}^{\infty} \xi(n) \alpha^{-n}\right)=\sum_{n=1}^{\infty} \xi(n) \beta^{-n} .
$$

Employing lemma 3.2 one finds that $\operatorname{dim} \mathscr{C}_{\alpha}$ depends only on $|\alpha|$ and that it forms a non-increasing function of $|\alpha|$. If $\alpha$ is a positive integer $>a$ then in view of $[3$, prop. III.1] $\operatorname{dim} \mathscr{C}_{\alpha}>0$. It follows that $\operatorname{dim} \mathscr{C}_{\lambda_{1}}>0$, and hence $\operatorname{dim} M_{2}>0$ also.

Turning to the general case let us suppose, for example, that $\left|\lambda_{i}\right|<1$ for $1 \leq i \leq k$ and $\left|\lambda_{i}\right|>1$ for $k+1 \leq i \leq s$. Similarly to the previous case we may assume each $\left|\lambda_{i}\right|$ 
to be either arbitrarily large or arbitrarily close to 0 . Select a positive integer $a$ with $4<a<\min _{1 \leq i \leq s}\left\{\max \left\{\left|\lambda_{i}\right|,\left|\lambda_{i}\right|^{-1}\right\}\right\}$ and set $\Delta=\{0,1, \ldots, a-1\}^{\mathbb{Z}}$. Let $T$ be the shift on $\Delta$. Making use of the set $M$ mentioned earlier one can construct a $T$-minimal set $M^{\prime} \subseteq \Delta$ having an exponential rate of growth of the blocks number. Put $u=$ $\sum_{i=k+1}^{s} v^{(i)}$. Define $\psi: \Delta \rightarrow \mathbb{R}^{r}$ by:

$$
\begin{aligned}
\psi(\xi) & =\sum_{n=-\infty}^{0} \xi(n) \sigma^{-n}(u-e)+\sum_{n=1}^{\infty} \xi(n) \sigma^{-n}(u) \\
& =-\sum_{i=1}^{k} \sum_{n=-\infty}^{0} \xi(n) \lambda_{i}^{-n} v^{(i)}+\sum_{i=k+1}^{s} \sum_{n=1}^{\infty} \xi(n) \lambda_{i}^{-n} v^{(i)} .
\end{aligned}
$$

The projection of $\psi\left(M^{\prime}\right)$ in $\nabla^{r}$ is $\sigma$-minimal and is shown to be of positive Hausdorff dimension precisely as in the former case. Thus the proof is complete.

\section{LEMMA 3.4. $\Sigma$ is multi-parameter.}

Proof. Suppose, to the contrary, that for some $v \in \operatorname{evec} \Sigma$ the semigroup $\operatorname{spec}_{v} \Sigma$ is weakly one-parameter. Set $K=\mathbb{Q}\left(\operatorname{spec}_{v} \Sigma\right)$. Consider the conjugates $v^{(1)}=v$, $v^{(2)}, \ldots, v^{(s)}$ of $v$ over $\mathbb{Q}$. These vectors are linearly independent. In fact, select a $\lambda \in K$ with $K=\mathbb{Q}(\lambda)$. There exists a matrix $\tau$ in the algebra $\Sigma^{\prime}$ generated by $\Sigma$ over $\mathbb{Q}$ such that $\tau v=\lambda v$. The vectors $v^{(1)}, v^{(2)}, \ldots, v^{(s)}$ form common eigenvectors of $\Sigma$, whence of $\Sigma^{\prime}$ as well. The eigenvalues $\lambda_{1}=\lambda, \lambda_{2}, \ldots, \lambda_{s}$ of $\tau$ corresponding to these eigenvectors are distinct, so that the latter are linearly independent. Put $V=\operatorname{sp}\left\{v^{(i)} \mid 1 \leq i \leq s\right\}$ and $e=\sum_{i=1}^{s} v^{(i)} . V$ is a $\sum$-invariant subspace of $\mathbb{C}^{r}$, and therefore $V_{\mathbb{R}}=V \cap \mathbb{R}^{r}$ and $V_{\mathbb{Z}}=V \cap \mathbb{Z}^{r}$ are $\Sigma$-invariant also. The vectors $e, \tau e, \ldots, \tau^{s-1} e$ are linearly independent, which implies that $V_{\mathbb{R}}$ is an $s$-dimensional subspace of $\mathbb{R}^{r}$ and $V_{\mathbb{Z}}$ is a free abelian group of rank $s$. It follows that $V_{\mathbb{R}} / V_{\mathbb{Z}}$ is a $\Sigma$-invariant closed subgroup of $\mathbb{T}^{r}$ isomorphic with $\mathbb{T}^{s}$. Limiting our attention to this subgroup we observe that it may be assumed, to begin with, that the conjugates of $v$ span $\mathbb{C}^{r}$, that is $v^{(1)}, v^{(2)}, \ldots, v^{(r)}$ are the conjugates of $v$.

Since $\Sigma$ is ergodic there exists an ergodic $\sigma \in \Sigma$ [2, th. 5.1]. Given any $\tau \in \Sigma$ denote by $\lambda_{1,}, \lambda_{2 \pi}, \ldots, \lambda_{r r}$ its eigenvalues corresponding to the basis $v^{(1)}, v^{(2)}, \ldots, v^{(r)}$. Our assumption concerning $\operatorname{spec}_{v} \Sigma$ means that for any $\tau \in \Sigma$ we have $\lambda_{1 \tau}^{l}=\lambda_{1 \sigma}^{m}$ for appropriately chosen non-zero $l, m \in \mathbb{Z}$. By conjugation similar equalities hold for the other eigenvalues, which leads to $\tau^{l}=\sigma^{m}$. The proof of proposition 3.2 in [1] implies the existence of finitely many epimorphisms $\sigma_{1}, \sigma_{2}, \ldots, \sigma_{k}$ of $\mathbb{T}^{r}$, each commuting with $\Sigma$, such that $\Sigma \subseteq \bigcup_{i=1}^{k} G(\sigma) \sigma_{i}$ or $\Sigma \subseteq \bigcup_{i=1}^{k} S(\sigma) \sigma_{i}$, depending on $\sigma$ being invertible or not, respectively. Take a $\sigma$-minimal set $M$ with $\operatorname{dim} M>0$. Let $M_{1}$ be any $\Sigma$-minimal subset of $\overline{\Sigma M}$. For a suitable $i$ we have $\sigma_{i}(M) \subseteq M_{1}$. Since ker $\sigma_{i}$ is finite [1, lemma 5.1], lemma 3.2 gives $\operatorname{dim} \sigma_{i}(M)>0$, and so certainly $\operatorname{dim} M_{1}>0$. This proves the lemma.

The implication $(3) \Rightarrow(4)$ in theorem 2.1 is thus proved.

\section{The sufficiency of the conditions}

This section is devoted to proving the implication $(4) \Rightarrow(1)$ in theorem 2.1. A special case will be established first. Given a matrix $\sigma$, its characteristic polynomial is 
denoted by $f_{\sigma}$. A commutative semigroup $\Sigma$ is called totally irreducible if there exists a $\sigma \in \Sigma$ with $f_{\sigma^{n}}$ irreducible over $\mathbb{Z}$ for every positive integer $n$.

Proposition 4.1. A totally irreducible hyperbolic multi-parameter commutative semigroup is $M T$.

Proof. Let $\Sigma$ be a semigroup having the described properties. In view of $[1$, lemma 3.4] there exists a basis of $\mathbb{C}^{r}$ consisting of common eigenvectors of $\Sigma$. It is easy to see that $\Sigma$ contains a finitely-generated subsemigroup satisfying the conditions of the proposition, so $\Sigma$ itself may be assumed to be finitely-generated. These conditions also secure the existence of an ergodic $\sigma \in \Sigma$. Proposition IV.1 of [3] can therefore be applied to conclude that if $M$ is any $\Sigma$-minimal set then $M-M$ is a proper subset of $\mathbb{T}^{r}$.

Decompose $\mathbb{C}^{r}$ in the form $\mathbb{C}^{r}=V_{\leq 1} \oplus V_{>1}$, where $V_{\leq 1}$ is the subspace of $\mathbb{C}^{r}$ spanned by those $v \in \operatorname{evec} \Sigma$ with $\operatorname{spec}_{v} \Sigma \subseteq\{z \in \mathbb{C}|| z \mid \leq 1\}$, and $V_{>1}$ is the subspace spanned by all the other common eigenvectors of $\Sigma$.

Assume that $\Sigma$ is not an $M T$ semigroup. Let $M$ be a $\Sigma$-minimal set composed of non-torsion elements. Since $\Sigma$ is ergodic $M$ is infinite. The set $M-M$ (or rather its lifting to $\mathbb{R}^{r}$, considered as a subset of $\mathbb{C}^{r}$ ) is a closed $\Sigma$-invariant set containing 0 as a non-isolated point. Assume first that $M-M$ contains a sequence converging to 0 of points with non-zero components in $V_{>1}$. The same construction as the one carried out in [1, lemma 4.2] supplies a sequence of non-zero points of $M-M$ lying in $V_{>1}$. Careful inspection of $\S 4$ of [1], from (4.1) on, reveals that these assumptions lead to $M-M=\mathbb{T}^{r}$, which is a contradiction.

It follows consequently that in a sufficiently small neighbourhood of 0 every point of $M-M$ lies in $V_{\leq 1}$. Select an ergodic $\sigma \in \Sigma$ and a $\sigma$-minimal set $M_{1} \subseteq M$. Take any $x \in M_{1}$. For an appropriately chosen sequence $\left(n_{k}\right)_{k=1}^{\infty}$ of positive integers $\sigma^{n_{k}} x \rightarrow_{k \rightarrow \infty} x$ (in $\mathbb{J}^{r}$ ). Hence there exists an $n$ such that

$$
\sigma^{n} x-x=\gamma+v, \quad \gamma \in \mathbb{Z}^{r}, v \in V_{\leq 1} .
$$

The definition of $V_{\leq 1}$ and the hyperbolicity of $\Sigma$ imply the existence of a $\tau \in \Sigma$ each of whose eigenvalues corresponding to common eigenvectors of $\Sigma$ lying in $V_{\leq 1}$ is of norm strictly less than 1 . Take a sequence $\left(n_{k}\right)_{k=1}^{\infty}$ with $\left(\tau^{n_{k}} x\right)_{k=1}^{\infty}$ convergent in $\mathbb{T}^{r}$. Letting $\tau^{n_{k}}$ act on both sides of (4.1) and passing to the limit in $\mathbb{T}^{r}$ we obtain $\sigma^{n} x^{\prime}=x^{\prime}$ for some $x^{\prime} \in M$. By [1, lemma 5.2] $x^{\prime}$ is a torsion element of $\pi^{r}$, which completes the proof.

It has to be shown that the assumption concerning total irreducibility in the proposition can be dropped. The following lemma forms the key to this.

LEMMA 4.1. Let $\Sigma$ be a semigroup of endomorphisms of $\pi^{r}$. Suppose $H$ is a closed $\Sigma$-invariant subgroup of $\pi^{r} . \Sigma$ is an MT semigroup iff the semigroups induced by $\Sigma$ on $H$ and on $\mathbb{T}^{r} / H$ are both $M T$ semigroups.

The proof is straightforward.

The reduction of the proof from the general case to that of totally irreducible semigroups will be carried out in two steps. Denote by $A$ the field of algebraic numbers. 
Step 1. It may be assumed that $A^{r}$ has a basis $v^{(1)}, v^{(2)}, \ldots, v^{(r)}$ composed of common eigenvectors of $\Sigma$ which are conjugate over $\mathbb{Q}$.

To accomplish this reduction it is needed, given any commutative semigroup $\Sigma$, to construct a basis of $\mathbb{A}^{r}$ with respect to which the action of $\Sigma$ is as simple as possible. For any function $\lambda: \Sigma \rightarrow \mathbb{A}$ set:

$$
U_{\lambda k}=\left\{v \in \mathbb{A}^{r} \mid \prod_{i=1}^{k}\left(\sigma_{i}-\lambda\left(\sigma_{i}\right)\right) v=0 \quad \forall \sigma_{1}, \sigma_{2}, \ldots, \sigma_{k} \in \Sigma\right\}, \quad k=0,1,2, \ldots
$$

The sequence $\{0\}=U_{\lambda_{0}} \subseteq U_{\lambda_{1}} \subseteq \cdots$ is formed of $\Sigma$-invariant subspaces of $\mathbb{A}^{r}$ and is constant from the $r$ th place on. Using the Jordan decomposition it is easy to verify that $A^{r}=\bigoplus_{\lambda \in A^{\Sigma}} U_{\lambda r}$. Take an arbitrary $\lambda_{1}: \Sigma \rightarrow A$ such that $U_{\lambda_{1} r} \neq\{0\}$. Let $K_{\lambda_{1}}=\mathbb{Q}\left(\lambda_{1}(\Sigma)\right)$. Select a basis of $U_{\lambda_{1} 1}$ consisting of vectors in $K_{\lambda_{1}}^{r}$, complete it to a basis of $U_{\lambda_{1} 2}$, complete this to a basis of $U_{\lambda_{1} 3}$ and so forth. We now have a basis of $U_{\lambda_{1} r}$ formed of vectors in $K_{\lambda_{1}}^{r}$. The conjugations of $K_{\lambda_{1}}$ give rise to conjugates of $\lambda_{1}$ and corresponding conjugates of the basis vectors of $U_{\lambda_{1} r}$. If the union of all these bases contains less than $r$ vectors then we continue by picking another function $\lambda_{2}: \Sigma \rightarrow A$ and following an analogous procedure. The process terminates after finitely many steps, giving a basis of $A^{r}$. This basis is of the form

$$
\left\{v_{i j k l} \mid 1 \leq i \leq s_{1}, 1 \leq j \leq s_{2}(i), 1 \leq k \leq s_{3}(i), 1 \leq l \leq s_{4}(i, k)\right\},
$$

where $s_{1}$ is the number of distinct non-conjugate functions $\lambda_{1}, \lambda_{2}, \ldots, \lambda_{s_{1}} \in \mathbb{A}^{\Sigma}$ with $U_{\lambda_{i} r} \neq\{0\}, s_{2}(i)$ is the number of conjugates of $\lambda_{i}, s_{3}(i)$ is the least positive integer $m$ such that $U_{\lambda_{i}, m+1}=U_{\lambda_{i}, m}$ and $s_{4}(i, k)=\operatorname{dim} U_{\lambda_{i}, k}-\operatorname{dim} U_{\lambda_{i}, k-1}$.

Let $V$ denote the subspace of $\mathbb{C}^{r}$ spanned by $\left\{v_{111} \mid l \leq l \leq s_{4}(1,1)\right\}$. Put $V_{R}=V \cap \mathbb{R}^{r}$, $V_{Z}=V \cap \mathbb{Z}^{r}$ and $r_{1}=s_{4}(1,1)$. One can show that $V_{\mathbb{R}}$ is an $r_{1}$-dimensional vector space over $\mathbb{R}$ and $V_{Z}$ is a discrete free abelian group of rank $r_{1}$. Now consider diagram 1 . The solid arrows represent naturally defined continuous homomorphisms. It is easy to check that the rows and columns marked by solid arrows form exact sequences. According to the $3 \times 3$ lemma [7, p. 172] the dashed arrows stand for uniquely determined homomorphisms which make the fourth row an exact sequence and the diagram commutative. The semigroup $\Sigma$ naturally gives rise to commutative semigroups of epimorphisms of $V_{\mathbf{R}} / V_{\mathbf{Z}}$ and of $\left(\mathbb{R}^{r} / V_{\mathbf{R}}\right) /\left(\mathbb{Z}^{r} / V_{\mathbb{Z}}\right)$. It is evident from the construction that if $\Sigma^{\prime}$ is either of these semigroups and $v \in \operatorname{evec} \Sigma^{\prime}$ then $\operatorname{spec}_{v} \Sigma^{\prime}=$ $\operatorname{spec}_{v_{1}} \Sigma$ for a suitably chosen $v_{1} \in \operatorname{evec} \Sigma$. Hence if $\Sigma$ is a hyperbolic multi-parameter semigroup then each of these induced semigroups is such. In view of lemma 4.1 it suffices to prove that each of them is $M T$. Repeated use of the decomposition process gives therefore the desired reduction.

Step 2. $\Sigma$ may be assumed to be totally irreducible.

In fact, let $v^{(1)}, v^{(2)}, \ldots, v^{(r)}$ be a basis of $\mathbb{A}^{r}$ having the properties described in the former step. The eigenvalues of any $\sigma \in \Sigma$ with respect to this basis are denoted by $\lambda_{1 \sigma}, \lambda_{2 \sigma}, \ldots, \lambda_{r \sigma}$ Each $\sigma \in \Sigma$ is uniquely determined by $\lambda_{1 \sigma}$. Put $K=\mathbb{Q}\left(\operatorname{spec}_{v}{ }^{(1)} \Sigma\right)$. Assuming that $\Sigma$ is not totally reducible we find that for every $\sigma \in \Sigma$ there exists an $n$ for which $\mathbb{Q}\left(\lambda_{1 \sigma}^{n}\right)$ is a proper subfield of $K$. Now we need

LEMMA 4.2. Let $K$ be a number field and $S$ a subsemigroup of the multiplicative group $K^{*}$ of $K$. Suppose that for every $s \in S$ there exists an $n \in \mathbb{N}$ such that $\mathbb{Q}\left(s^{n}\right)$ is a proper 


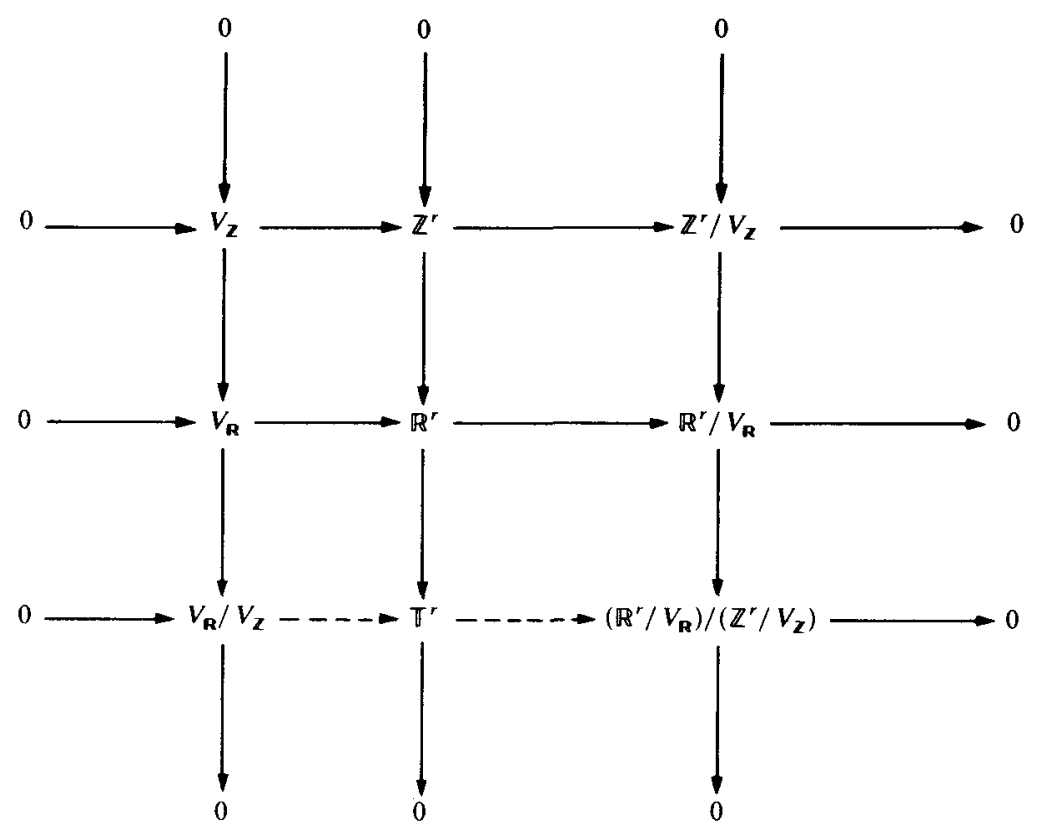

DIAGRAM 1

subfield of $K$. Then there exists $n \in \mathbb{N}$ and a proper subfield $F$ of $K$ such that $s^{n} \in F$ for every $s \in S$.

Proof. Put $F(s)=\bigcap_{n=1}^{\infty} \mathbb{Q}\left(s^{n}\right), s \in S$. Let us show first that if $F\left(s_{1}\right)=F_{1}$ and $F\left(s_{2}\right)=F_{2}$ for some $s_{1}, s_{2} \in S$ then $F(s)=\mathbb{Q}\left(F_{1} \cup F_{2}\right)$ for an appropriately chosen $s \in S$. In fact, the correspondence $(m, n) \rightarrow F\left(s_{1}^{m} s_{2}^{n}\right)$ gives a finite colouring of $\mathbb{N} \times \mathbb{N}$. By Grünwald's theorem concerning finite colourings of $\mathbb{N} \times \mathbb{N}$ (see, for example, [5, th. 0.6]) there exist $m_{1}, m_{2}, n_{1}, n_{2} \in \mathbb{N}, m_{1}<m_{2}, n_{1}<n_{2}$, with $F\left(s_{1}^{m_{1}} s_{2}^{n_{1}}\right)=F\left(s_{1}^{m_{2}} s_{2}^{n_{1}}\right)=F\left(s_{1}^{m_{1}} s_{2}^{n_{2}}\right)$. Since $F\left(s^{n}\right)=F(s)$ for every $s \in S$ and $n \in \mathbb{N}$, we may assume that $F\left(s_{1}^{m} s_{2}^{n}\right)=\mathbb{Q}\left(s_{1}^{m} s_{2}^{n}\right)$ for $(m, n)=\left(m_{1}, n_{1}\right),\left(m_{2}, n_{1}\right),\left(m_{1}, n_{2}\right)$. This implies $s_{1}^{m_{2}-m_{1}}, s_{2}^{n_{2}-n_{1}} \in F\left(s_{1}^{m_{1}} s_{2}^{n_{1}}\right)$, which leads to $F\left(s_{1}\right), F\left(s_{2}\right) \subseteq F\left(s_{1}^{m_{1}} s_{2}^{n_{1}}\right)$. Thus $s=s_{1}^{m_{1}} s_{2}^{n_{1}}$ is an element of $S$ possessing the required property.

The conditions of the lemma now mean that $F=\mathbb{Q}\left(\bigcup_{s \in S} F(s)\right)$ is a proper subfield of $K$. For any subset $A$ of $K$ let

$$
\sqrt{A}=\left\{\alpha \in K \mid \exists n \in \mathbb{N} ; \alpha^{n} \in A\right\} \text {. }
$$

With this notation we have $S \subseteq \sqrt{F}$. From the proof of proposition 3.1 in [1] we infer that $\sqrt{F^{*}}=\bigcup_{i=1}^{m} F^{*} \alpha_{i}$ for suitable $\alpha_{1}, \alpha_{2}, \ldots, \alpha_{m} \in \sqrt{F^{*}}$. This implies that for some $n \in \mathbb{N}$ we have $\alpha^{n} \in F^{*}$ for every $\alpha \in \sqrt{F^{*}}$, and in particular for every $\alpha \in S$. This proves the lemma.

Returning to our reduction step, we find that there exists a positive integer $n$ and a proper subfield $F$ of $K$ such that $\lambda_{1 \sigma}^{n} \in F$ for every $\sigma \in \Sigma$. Denote by $\Sigma^{\prime}$ the subsemigroup of $\Sigma$ generated by $\left\{\sigma^{n} \mid \sigma \in \Sigma\right\}$. $\Sigma^{\prime}$ is evidently a hyperbolic multiparameter semigroup if $\Sigma$ is. To $\Sigma^{\prime}$ we can now apply the decomposition process carried 
out in the first reduction step. Repeated alternating use of the processes described in this and the former step lead us at last to a situation where all involved semigroups are totally irreducible, in addition to being hyperbolic and multi-parameter.

The last reduction step and proposition 4.1 complete the proof of theorem 2.1 .

\section{REFERENCES}

[1] D. Berend. Multi-invariant sets on tori. Trans. Amer. Math. Soc. 280 (1983), 509-532.

[2] D. Berend. Ergodic semigroups of epimorphisms. Trans. Amer. Math. Soc. (To appear.)

[3] H. Furstenberg. Disjointness in ergodic theory, minimal sets and a problem in diophantine approximation. Math. Systems Theory 1 (1967), 1-49.

[4] H. Furstenberg. Intersections of Cantor sets and transversality of semigroups. In Problems in Analysis, (R. C. Gunning - General editor). Princeton University Press: Princeton, New Jersey, 1970, p. 41-59.

[5] H. Furstenberg \& B. Weiss. Topological dynamics and combinatorial number theory. J. d'Analyse Math. 34 (1978), 61-85.

[6] G. H. Hardy \& J. E. Littlewood. The fractional part of $n^{k} \theta$. Acta. Math. 37 (1914), 155-191.

[7] B. Pareigis. Categories and Functors. Academic Press: New York and London, 1970. 